\title{
Science of cryopreservation in reproductive medicine - Embryos and oocytes as exemplars
}

Taisiia Yurchuk a, b, Ph. D, Maryna Petrushko a, b, D.Sc, Barry Fuller c, D. Sc.

a Institute for Problems of Cryobiology and Cryomedicine of the National Academy of Sciences of Ukraine \& UNESCO Chair in cryobiology, Ukraine

b ART-clinic for human reproduction, Ukraine

c Divison of Surgery \& Interventional Science, Royal Free London NHS Trust \& UCL, Royal Free Campus, London $N W 32 Q G, U K$

Taisiia Yurchuk, e- mail:taisiya.yur@gmail.com Tel: +380934591320

Maryna Petrushko,e-mail: petrushkomarina@gmail.com

Barry Fuller,e-mail:b.fuller@ucl.ac.uk

Correspondence: Taisiia Yurchuk, PhD Institute for Problems of Cryobiology and Cryomedicine of the National Academy of Sciences of Ukraine \& UNESCO Chair in cryobiology, 23, Pereyaslavskaya str., Kharkov, Ukraine 61015, Tel: +380 57373 4143, Fax: +380 57373 3084; ART-clinic for human reproduction, 38 B Gagarina av.,Kharkiv, Ukraine; e- mail:taisiya.yur@gmail.com Tel: +380934591320

\section{ABSTRACT}

The modern successes of reproductive medicine are based on the achievements in the fields of artificial fertilization and cryobiology over the last 50 years. Cryopreservation of oocytes makes it possible to preserve their reproductive potential after surgical interventions, treatment of cancer, for delayed pregnancy and to use cells for donation. Cryopreservation of embryos allows not only to reduce the multiple pregnancies rate and to in- crease the cumulative pregnancy rate as a result of embryo transfer in the following favorable cycles of the patient, but is also a necessary procedure in case of genetic diagnosis or in the case of contraindications for embryo transfer in the stimulated cycle due to possible complications. However, the viability of cryopreserved oocytes and embryos depends on the degree of their cryo damage during the process of freeze-warming. In this regard, it is very important to develop such freezing protocols that minimize the damages caused by the intra- and extracellular ice crystal formation, toxic effect of high concentrations of cryoprotectants and osmotic stresses. The effectiveness of cryopreservation of gametes and embryos is assessed on the basis of morphological, functional and genetic changes in the cells after warming. Special attention should be paid to the ethical issues of assisted reproductive technology, including cryobiotech technologies, which in many countries remain open and in need of settlement.

\section{Introduction}

New opportunities in the treatment of infertility by methods of assisted reproductive technologies (ART), which increase the cumulative incidence of pregnancy, appeared after it became possible to preserve the unique reproductive material by ultra-low-temperature preservation. Successful cryopreservation of reproductive cells is possible under condition of knowledge as the fundamental principles of cryobiology covering the course of physical and chemical processes and individual biological properties of the object.

2. History of gametes and human embryos cryopreservation

In 1964, a group of scientists founded the International Society of Low-Temperature Biology, whose goal was to contribute to the study of the effect of low temperatures on all types of organisms, organs, tissues 
and cells [1]. At about the same time, in USA, the Society for Cryobiology was formed, and early members included P Mazur and S Leibo, who were pioneers of reproductive cryobiology. In the 60s and 70 s of the last century they determined the permeability characteristics of membranes of different cell types for water and cryoprotectant solutions. The two-factor theory of cryodamages advanced by P. Mazur in 1972 became the biophysical basis of experiments on gametes and human embryos cryopreservation [2]. Fundamental research at this time, was aimed at elucidating the mechanism of freezing action on cells, namely: the study of the permeability of membranes for water and cryoprotectants, the osmotic behavior of cells in solutions of cryoprotectants, the effect of physicochemical factors of cell damage and cryoprotection [3]. It became clear that the protocols that were optimal for one cell type are ineffective for others and are species-specific. In the 1960s, English doctor-gynecologist P. Steptoe and embryologist R. Edwards began joint research and put forward an idea, the essence of which was to help infertile couples give birth to a child [4]. However, most of their colleagues believed that the use of spermatozoa and human eggs was unethical and immoral. Scientists were denied state funding research. However, the problem of sterile marriage in the UK acquired growing interest. Women voluntarily participated in the research, with informed consent signing documents on the governance of the assisted reproductive technologies (ART) cycles.

In 1976 a significant event occurred: after the oocyte in vitro fertilization scientists managed to achieve the first pregnancy which turned out to be ectopic. In 1977, a group of researchers led by P. Steptoe conducted a successful treatment cycle the result of which was the world's first child "from a test tube birth" on July 25, 1978. Since then, the number of children born through in vitro fertilization methods has been steadily increasing every year. According to modern statistics conception $2 \%$ of newborns have occurred by IVF [5]. The contribution of these technologies to the world's scientific potential was recognized by Dr. R. Edwards awarding with the Nobel Prize in 2010 in the field of physiology and medicine.

For a long time attempts to cryopreserve human oocytes were doomed to failure which can be explained by the lack of detailed knowledge of the structure of female sexual gametes. The first attempt at lowtemperature storage of mammalian oocytes is attributed to J. Sherman and T. Lin (1959) who froze mouse oocytes to $-10{ }^{\circ} \mathrm{C}$ using glycerol. The first the birth of live mice after the storage of oocytes in liquid nitrogen was documented in 1977 by D. Whittingham. In 1986 it was reported about the childbirth after oocytes freezing by slow cooling, but because of very low effectiveness of this method (achieving only five pregnancies over a decade of experience) the experimental work was discontinued [6].

In 1991 a method for injecting a sperm into the cytoplasm of the oocyte (ICSI) performed under a microscope using multiple micromanipulation devices was developed at the University of Bruxelles. In 1997 after thawing oocytes was successfully fertilized by ICSI. Using this method allowed to increase the frequency rate of oocyte fertilization [7].

The first report in the world to successfully vitrify donor oocytes and claim pregnancy in a 47-year-old woman after transferring the embryo, obtained from a thawed oocyte was made in 1999 [8].

The history of the development and use of cryopreservation of embryos proved to be rather complicated because of moral, ethical, legal and religious contradictions arose. The first pregnancy resulting from transfer of a thawed cryopreserved human embryo was reported in 1983 in Australia [9], and the first live birth following embryo cryopreservation was reported in 1984 in The Netherlands [10]. The ultrafast mode of freezing during cryopreservation of human embryos was first introduced in 1987 [11]. In 1995 the first child was born after cryopreservation of blastocysts and in 1996 it was possible to cryopreserve an embryo obtained in the natural cycle of a woman before chemotherapy [12]. Currently $>50,000$ children have been born from the frozen embryos which have been stored for a long time in liquid nitrogen and subsequently thawed. However it is important not only to obtain the high survival rate of cryopreserved reproductive cells and embryos but also to guarantee the biosafety of cryotechnologies. The development and implementation of pre-implantation genetic diagnosis (PGD) made it a selective tool for determining embryos with normal karyotypes [13]. This practice is called "pre-implantation genetic screening" (PGS). In the 80-90's XXth century it was at once suggested different methods of biopsy of blastomeres, polar body and trophectoderm for PGD and PGS. In 2006 the first successful 
pregnancy was obtained after preimplantation genetic diagnosis of aneuploidies followed by cryopreservation of embryos [14].

\section{Medical and social indications for cryopreservation of oocytes and embryos}

The need to develop reproductive cryotechnology is due to the many situations that women face during their lifetime. Among them there is an increase number of cancer patients of reproductive age. After a relapse-free treatment of this pathology for five years, a woman can give birth to a child using oocytes, which were prudently preserved in the cryobank. Moreover, oocyte cryopreservation can be included in the treatment standards of such patients.

In ART programs, cryopreserved oocytes are used in cases of ovarian hyperstimulation syndrome [15]. Indications for the cryopreservation of oocytes are genetic risks: the status of carriers of the BRCA mutation in- stability of the selection of the X chromosome, Turner's syndrome [16]. Also the evidence for the fertility preservation is a hereditary predisposition to the early menopause.

Sometimes oocytes may be cryopreserved in case of force majeure, when for some reason it is not possible to get partner sperm on the day of oocyte aspiration. The successful results of cryopreservation of female gametes optimize the program of their donation. Practice of oocytes donation obtained in synchronized fashion with the donor menstrual cycle takes time. Cryopreservation gives time in general for genetic and infectious screening of donor reproductive cells.

Recently, social indicators for oocyte cryopreservation have become increasingly popular, as more women want to give birth to a child only after certain achievements in their life or career sphere. However, the patient should be warned about the low pregnancy rates by in the case of social cryopreservation of female sex gametes.

In contrast to eggs preservation, embryos freeze-thawing for further transfer to the uterine cavity allows to obtain very high results of their viability and to increase the cumulative incidence of pregnancy by approximately $10 \%$ [17].

Priorities for human reproductive clinics is avoiding the multiple pregnancy complication and complications as a result of endometrial bleeding, increased progesterone level or other unplanned risks after embryo transfer delay. One way to achieve this target is a single embryo transfer to the patient and cryopreservation of the remaining ones to use them in further cycles. Also embryo cryopreservation allows to preserve the reproductive potential of patients before surgical interventions on pelvic organs.

Many clinics accept for these reasons the practice of "freeze all" in such cases as the ovarian hyperstimulation syndrome which can cause negative consequences after the fresh embryos transfer or for carrying out PGD in case of possible appearance of hereditary genetic diseases in children. A recent study showed an increased risk of gestational age and high birth weight associated with pregnancy as a result of the thawed embryo transfer. This should be taken into account when adopting a pol- icy for the elective freezing of all embryos in IVF [18].

\section{Cryopreservation features of oocytes and preimplantation embryos}

There is an assumption that the main damage in the cryopreservation of oocytes and embryos occurs results from on the one hand of ice crystals action of during the freezing and recrystallization process at the thawing and osmotic stress which occurs during exposure and removal of cryoprotective solutions on the other. In this regard to ensure the cell survival to cryoprotectants agents (CPA) are used, osmotic and chemical toxicities should be considered. [19]

Advances in the field of cryopreservation of gametes and embryos associated with the discovery and subsequent development of the con- cept of cryoprotectant agents. Depending on their interactions with 
the biological samples the cryoprotectants are divided into two groups: penetrating and non-penetrating. Penetrating cryoprotectants reduce the freezing point of the solution, interact with the membrane structures of the cell, prevent high concentrations of intra- and extracellular electrolytes, and partially replace water. Dimethylsulfoxide (DMSO), ethylene glycol (EG), glycerol, formamide, methanol and propylene glycol are the most commonly used for freezing human reproductive cells and embryos. Other penetrating substances, such as urea, acetamide, $\mathrm{N}$-methylformamide, diethylene glycol, triethylene glycol, n-propanol, isopropanol, 1,3-propanediol, 1,3-butanediol, 2-methoxyethanol and 3-methoxy-1, 2 propanediol have limited cryoprotective properties [19]. The principle of the action of non-penetrating cryoprotectants is not fully understood. Probably, it consists in reducing the growth rate of crystals and protecting the cell from osmotic changes. Non-penetrating cryoprotectants include two groups of substances: oligosaccharides (su- crose and trehalose are most often used) and high-molecular compounds (ficoll, albumin, polyvinylpyrrolidone etc.).

Sucrose and trehalose are the most common non-penetrating cryoprotectants used for vitrification of human embryos and oocytes [20]. The presence of sucrose in a solvent sharply reduces the susceptibility of oocytes and embryos to osmotic shock.

Penetrating cryoprotectants are becoming more toxic as their con- centration increases [21]. The toxic effect on the cells can be reduced by using a mixture of CPAs, where the proportion of each protective agent will be reduced, but the total concentration will remain the same. To achieve the oocyte/embryo glassy state during freezing - vitrification, high concentrations of CPAs and cooling rates are used to avoid ice for- mation. On the other hand, such agents possess high osmolality and various osmotic stress reduction procedures, such as rigorous compliance with the solution exposure time and an increase in washing steps after thawing are used to avoid the osmotic stress of oocytes and embryos. To date there is no consensus on the choice of a universal cryoprotectant for a particular cell.

The programs that are used for cryopreservation are traditionally divided into groups, depending on the rates of cooling of the bio-materi- als: controlled slow (0.1-5 deg./min), fast (10-100 deg./min) and vitrification, at which ultrafast velocities develop (more 10,000 deg./min). To achieve a high cooling rate, small volumes of cryoprotecting medium must be used [22].

To meet these requirements, it is necessary to select carriers that are capable of providing such high speeds when they are immersed in liquid nitrogen. Various systems are used, which can be divided into two groups: open and closed.

Controlled slow freezing, as a rule, uses closed media (straws, cryo-tubes). Open media are used mainly for vitrification - e.g. Cryoloop is a nylon loop, Cryotop, Cryo-leaf-plastic, Cryotech-polypropylene container. Standard straw is a system that can be used both open and closed, if it is in middle of another straw ("Straw-in-straw"). It is believed that closed systems will avoid the contamination of reproductive material during exposure to liquid nitrogen [23].

The warming step is equally important for survival rate of cryopreserved samples. It depends on the cryopreservation container, the freezing method and the cryoprotectant used. The cryoprotectants removal is been achieved by minimizing the osmotic pressure on the cells using solutions with stepwise decreased concentrations of cryoprotectants.

Thus, all existing methods for oocytes and embryos freezing can be classified as mainly aimed at reducing damage from ice crystals or in a category that reduces osmotic stress during the freeze-thawing, important strategies for an effective freezing protocol take these two factors into account.

\section{Morphological and functional evaluation of cryopreserved oocytes and embryos}

It is known that the oocyte is the largest cell in the human body. The average isotonic volume of the oocyte is $2.65 \times 106 \mu \mathrm{m} 3$. Due to its spherical shape, it has a low ratio of surface area to volume $(<0.01)$. 
The properties of plasma membranes (relatively low hydraulic conductivity and membrane permeability for cryoprotectants) do not allow the use of standard protocols for oocyte freezing.

Mature oocytes are at the stage of the second meiotic division (M II), which is sensitive to physical and chemical influences in cryopreservation. The release of oocytes from surrounding cumulus cells and granulosa (denudation) improves the oocyte survival rate [24].

Usually a heterogeneous pool of oocytes are recovered which are at the different stages of meiosis after aspiration of the follicles in IVF treatment cycle. Human oocytes, which differ in their degree of maturity, have a different value of the permeability of the plasma membrane which must be taken in their freezewarming protocols development. During the in vitro oocyte culturing the nucleus maturation begins. How- ever, this does not mean that it always accompanied by cytoplasm maturation that impacts on further fertilization outcomes. That is why the oocyte morphological parameters must be taken into account before cryopreservation as a predictors of their cryoresistance: cytoplasmic - oocyte size, maturity of the nucleus, presence of the meiotic spindle of fission, granulation, vacuoles, smooth endoplasmic reticulum, lipofuscin ovitels and extracytoplasmic - appearance of the first polar body, the thickness of perivitelline space and Zona pellucida.

During cryopreservation oocytes are affected by physical and chemical factors which can damage the spindle separation, alter the function of mitochondria or cause ZP hardening, because of premature release of cortical granules, changes in the glycoprotein layer, which plays an important role in fertilization, since the spermatozoon must bind to the ZP receptors, merge with the plasma membrane and fertilize the oocyte [25]. That is why it is impossible to fertilize the oocytes with normal insemination and cryopreserved oocytes has not been used into clinical practice for a long time. Only improved fertilization procedure such as ICSI could overcome this problem.

The meiotic apparatus performs the function of chromosome segregation to daughter cells during mitosis, and also to the first and second polar bodies during meiosis. The oocyte cytoskeleton (including microtubules and associated microfilaments) is responsible for the formation of the polar body, pronuclei and cell division. The meiotic apparatus must be accurately operated to achieve the proper chromosome segregation and to avoid aneuploidy. However at subphysiological temperatures the microtubules that form the fission spindle tend to depolymerize its structure, which can negatively affect the future embryo quality [26]. Despite the high survival rate of cryopreserved oocytes, there is a risk of chromosomal aneuploidy or other karyotypic abnormalities and further research is needed to clarify this very important issue.

Currently in clinical practice, the evaluation of the embryo quality is usually carried out according to morphological characteristics by system of sequential embryo selection (SES).

This classification assesses the division rate of embryo, the symmetry of blastomeres, the degree of cytoplasmic fragmentation, and the multi- nucleation of blastomeres.

Despite the achievements of cryobiology in the effectiveness of embryo cryopreservation the optimal development stage for cryopreservation remains an open question. [27]. Although there are no differences in the survival rate after warming of 2, 4, 8-cell embryos, morulas and blastocysts, most practitioners believe that freezing of embryos at the stage of blastocyst is more promising, since it is better able to predict the embryo development in vitro. Embryos with developmental delay or cytoplasm fragmentation can be identified in advance, this will allow to cryopreserve only competent embryos.

However morphological assessment of embryo quality of cannot be a reliable indicator of their viability. First, not always embryo damages, even lethal, immediately manifest morphological changes. Thus, the tur- bidity of the cytoplasm and the nucleus contrast appear after the physiological disturbances proceeding to the degradation stage. Secondly, destruction of individual blastomeres does not prevent the further development of embryos. Each blastomer is capable of developing into blastocysts, because of their totipotency. 
Discussion remains around the issue of cryopreservation of human embryos with interventions on ZP. Biosy of ZP increases the permeability of the embryo membrane, and this can change the procedure of exposure with cryoprotectants and the process of dehydration.

The cryopreservation of human embryos, after biopsy of polar bodies or blastomeres for preimplantation genetic diagnosis, remains problematic. Biopsy of blastomeres after freezing-warming leads to better survival of embryos compared to biopsy prior to cryopreservation [28].

The embryo cryopreservation efficiency can be improved by prior assisted hatching, as this facilitates the cryoprotectant penetration and blastocoel dehydration. Expanded blastocysts in contrast to the early embryos may be more sensitive to cryopreservation since they are characterized by the presence of a large cavity - blastocoel filled with liquid. Under conditions of insufficient dehydration, the formation of ice crystals is possible in the cryopreservation process. There are various methods is carried out to reduce the blastocoel cavity prior the cryopreservation including mechanical, chemical and laser collapsing. However, at the present time the international standard of collapsing is not developed [29].

Despite the widespread use of cryotechnology in reproductive medicine, there are still concerns about the negative impact of cryopreservation factors on the embryogenetic apparatus, the potential organ defects or other developmental problems.

Special attention should be paid to the modern era of biotechnology application of embryo cryopreservation, e.g. PGS which prevents the transfer of a genetically infertile embryo to the uterine cavity. However, the survival rate of cryopreserved embryos after PGD is significantly lower than in blastomere/trophododerm free from frozen biopsies.

6. Ethical and legal aspects of cryopreservation of oocytes and embryos

Cryopreservation and further storage of human reproductive cells and embryos create prerequisites for the emergence of ethical and legal issues. The problem is that under the contract with the clinic, when the specified period of storage of gametes and embryos ends, the personnel of the clinic are responsible for their future. In European countries, a clear procedure for the separation of patients and physicians of this dilemma is developed. The family in writing, in an informed document, determines whether it needs to prolong storage for repeated pregnancy. According to the recommendations of ESHRE, reproductive cells and embryos should be stored and used in the reproductive period of patients, and their storage in cryobank is limited to 10 years [30]. The is- sue of the fate of reproductive cells and embryos, in the case of a patient's death, is discussed prior to the procedure of cryopreservation.

The question of whether to allow studies on reproductive cells and preimplantation embryos in vitro and if so, on what terms, is one of the most sensitive ethical issues that needs to be addressed. At the international level, it was agreed to observe a certain balance between the need to protect the embryo, on the one hand, and on the other the freedom of scientific research, [31].

Protective measures of embryos sacrificed for research purposes are primarily intended to ensure the proper nature of the research objectives and the content of embryos in appropriate conditions for as long as the defined study objective allows.

The development of policies and guidelines for working with cryopreserved materials should be based on the protection of the reproductive cells and embryos rights monitored by local ethical committees, patient representatives and expert associations.

\section{References}

[1] D.E. Pegg, The history and principles of cryopreservation, Semin. Reprod. Med. 20 (1) (2002) 513. 
[3] A. Arav, Cryopreservation of oocytes and embryos, Theriogenology 81 (1) (2014) 96-102.

[4] M.H. Johnson, Robert Edwards: the path to IVF, Reprod. BioMed. Online 23 (2) (2011) 245-262.

[5] M.J. Faddy, M.D. Gosden, R.G. Gosden, A demographic projection of the contribu- tion of assisted reproductive technologies to world population growth, Reprod. BioMed. Online 36 (4) (2018) 455-458.

[6] C. Chen, Pregnancy after human oocyte cryopreservation, Lancet 1 (8486) (1986) 884-886, (London, England).

[7] G. Palermo, H. Joris, P. Devroey, A.C. Van Steirteghem, Pregnancies after intracy- toplasmic injection of single spermatozoon into an oocyte, Lancet 340 (8810) (1992) 17-18, (London, England).

[8] L. Kuleshova, L. Gianaroli, C. Magli, A. Ferraretti, A. Trounson, Birth following vit- rification of a small number of human oocytes: case report, Hum. Reprod. 14 (12) (1999) 3077-3079, (Oxford, England).

[9] A. Trounson, L. Mohr, Human pregnancy following cryopreservation, thawing and transfer of an eight-cell embryo, Nature 305 (5936) (1983) 707-709.

[10] G.H. Zeilmaker, A.T. Alberda, I. van Gent, C.M. Rijkmans, A.C. Drogendijk, Two pregnancies following transfer of intact frozen-thawed embryos, Fertil. Steril. 42 (2) (1984) 293-296.

[11] A. Trounson, A. Peura, C. Kirby, Ultrarapid freezing: a new low-cost and effective method of embryo cryopreservation, Fertil. Steril. 48 (5) (1987) 843-850.

[12] J. Cohen, R.F. Simons, C.B. Fehilly, S.B. Fishel, R.G. Edwards, J. Hewitt, et al., Birth after replacement of hatching blastocyst cryopreserved at expanded blasto- cyst stage, Lancet 1 (8429) (1985) 647, (London, England).

[13] R.T. Scott Jr., K. Ferry, J. Su, X. Tao, K. Scott, N.R. Treff, Comprehensive chromo- some screening is highly predictive of the reproductive potential of human em- bryos: a prospective, blinded, nonselection study, Fertil. Steril. 97 (4) (2012) 870-875.

[14] M.C. Magli, L. Gianaroli, N. Grieco, E. Cefalu, G. Ruvolo, A.P. Ferraretti, Cryop- reservation of biopsied embryos at the blastocyst stage, Hum. Reprod. 21 (10) (2006) 2656-2660, (Oxford, England).

[15] E. Lucena, D.P. Bernal, C. Lucena, A. Rojas, A. Moran, A. Lucena, Successful ongo- ing pregnancies after vitrification of oocytes, Fertil. Steril. 85 (1) (2006) 108-111.

[16] E. Deligeoroglou, E. Stergioti, K.D. Dimopoulos, V. Karountzos, Y. Prapas, Preg- nancy outcome after oocyte donation in patients with Turner's syndrome: clinical experience and management, J. Obstet. Gynaecol. 36 (4) (2016) 504-507.

[17] M. Roque, M. Valle, A. Kostolias, M. Sampaio, S. Geber, Freeze-all cycle in repro- ductive medicine: current perspectives, JBRA Assist. Rep. 21 (1) (2017) 49-53.

[18] A. Maheshwari, S. Pandey, E. Amalraj Raja, A. Shetty, M. Hamilton, S. Bhat- tacharya, Is frozen embryo transfer better for mothers and babies? Can cumulative meta-analysis provide a definitive answer?, Hum. Reprod. Update 24 (1) (2018) 35-58.

[19] G.D. Elliott, S. Wang, B.J. Fuller, Cryoprotectants: a review of the actions and ap- plications of cryoprotective solutes that modulate cell recovery from ultra-low tem- peratures, Cryobiology 76 (2017) 74-91. 
[20] L.L. Kuleshova, D.R. MacFarlane, A.O. Trounson, J.M. Shaw, Sugars exert a major influence on the vitrification properties of ethylene glycol-based solutions and have low toxicity to embryos and oocytes, Cryobiology 38 (2) (1999) 119-130.

[21] B.P. Best, Cryoprotectant toxicity: facts, issues, and questions, Rejuvenation Res. 18 (5) (2015) 422-436.

[22] M. Kuwayama, Highly efficient vitrification for cryopreservation of human oocytes and embryos: the Cryotop method, Theriogenology 67 (1) (2007) 73-80.

[23] D.C. Joaquim, E.D. Borges, I.G.R. Viana, P.A. Navarro, A.A. Vireque, Risk of conta- mination of gametes and embryos during cryopreservation and measures to prevent cross-contamination, Biomed. Res. Int. 2017 (2017), 1840417.

[24] C. Patrat, A. Kaffel, L. Delaroche, J. Guibert, P. Jouannet, S. Epelboin, et al., Opti- mal timing for oocyte denudation and intracytoplasmic sperm injection, Obstet. Gynecol. Int. 2012 (2012), 403531.

[25] A. Jones, J. Van Blerkom, P. Davis, A.A. Toledo, Cryopreservation of metaphase II human oocytes effects mitochondrial membrane potential: implications for devel- opmental competence, Hum. Reprod. 19 (8) (2004) 1861-1866, (Oxford, England).

[26] L. Rienzi, C. Gracia, R. Maggiulli, A.R. Labarbera, D.J. Kaser, F.M. Ubaldi, et al., Oocyte, embryo and blastocyst cryopreservation in ART: systematic review and meta-analysis comparing slowfreezing versus vitrification to produce evidence for the development of global guidance, Hum. Reprod. Update 23 (2) (2017) 139-155.

[27] T. Mukaida, C. Oka, Vitrification of oocytes, embryos and blastocysts, Best Pract. Res. Clin. Obstet. Gynaecol. 26 (6) (2012) 789-803.

[28] S. Shinar, N. Kornecki, T. Schwartz, N. Mey-Raz, H. Amir, B. Almog, et al., Timing embryo biopsy for PGD - before or after cryopreservation?, Gynecol. Endocrinol. 32 (9) (2016) 756-758.

[29] E. Darwish, Y. Magdi, Artificial shrinkage of blastocoel using a laser pulse prior to vitrification improves clinical outcome, J. Assist. Reprod. Genet. 33 (4) (2016) 467-471.

[30] F. Shenfield, G. Pennings, C. Sureau, J. Cohen, P. Devroey, B. Tarlatzis, III. Gamete and embryo donation, Hum. Reprod. 17 (5) (2002) 1407-1408, (Oxford, England).

[31] B.M. Dickens, R.J. Cook, The legal status of in vitro embryos, Int. J. Gynaecol. Ob- stet. 111 (1) (2010) 91-94. 